\title{
Text-based classification incoming maintenance requests to maintenance type
}

\begin{abstract}
Classifying maintenance request is one of the important task in the large software system, yet often in large software system are not well classified. This is due to difficult in classifying by software maintainer. The categorization of maintenance type is effect on determine the corrective, adaptive, perfective, and preventive which are important to determine various quality factors of the system. In this paper we found that the requests for maintenance support could be classified correctly into corrective and adaptive. We used two different machine learning techniques alternatively Naïve Bayesian and Decision tree to classify issues into two type. Machine learning approach used the features that could be effective in increasing the accuracy of the system. We used 10-fold cross validation to evaluate the system performance. 1700 issues from shipment monitoring system were used to asses the accuracy of the system.
\end{abstract}

Keyword: Software maintenance; Maintenance type; Classification 